\title{
Cancer Data Standards Repository
}

National Cancer Institute

\section{Source}

National Cancer Institute. Cancer Data Standards Repository. NCI Thesaurus. Code C80150.

A database and a set of APIs and tools used to create, edit, control, deploy and find common data elements (CDEs) for metadata consumers and for UML model development. 